\title{
Prof. Andrzej Wiśniewski - FNP Prize Laureate 2019
}

DOI: http://dx.doi.org/10.12775/ZN.2019.020

\begin{abstract}
Prof. Andrzej Wiśniewski from the Faculty of Psychology and Cognitive Science at Adam Mickiewicz University in Poznań has received the 2019 Foundation for Polish Science Prize in the field of the humanities and social sciences for developing the concept of inferential erotetic logic.
\end{abstract}

Andrzej Wiśniewski was born in 1958 in Poznań. In 1981 he earned a master's degree in philosophy at Adam Mickiewicz University. After his graduation, he joined the Institute of Philosophy at the university, where he completed his doctorate in 1986. He earned his postdoctoral (habilitation) degree in 1991 with a dissertation entitled The Posing of Questions: Logic and Rationality. He was awarded full professorship in 1999.

In 1995 he began working at the Tadeusz Kotarbiński Pedagogical University in Zielona Góra, where he subsequently headed the Institute of Philosophy and in 1999 was elected rector. He served in this position until 2001, when the institution merged with the Technical University of Zielona Góra to form the University of Zielona Góra. He worked at the Institute of Philosophy at the University of Zielona Góra until 2005, when he moved to the Department of Logic and Cognitive Science at Adam Mickiewicz University in Poznań, which he headed until 2007 and where he still works.

Prof. Wiśniewski has also conducted research at many foreign institutions, including the University of California, Riverside, the Royal Flemish Academy of Belgium for Science and the Arts in Brussels, the Netherlands Institute for Advanced Study in the Humanities and Social Sciences in Wassenaar, and the University of Cambridge.

He is a laureate of the MISTRZ/MASTER programme of the Foundation for Polish Science and the Maestro programme of the National Science Centre (NCN). He has also been recognized for his accomplishments by the Prize of the Minister of National Education, the Prize of the Minister of Science and Higher Education, the Gold Cross of Merit, and the Knight's Cross of the Order of Polonia Restituta.

Prof. Wiśniewski has presented his results in five monographs, the latest of which is Questions, Inferences, and Scenarios (London: College Publications, 2013). He has also published some 50 papers, most of them in prestigious international journals on logic and analytical philosophy. 
The 2019 Foundation for Polish Science Prize honours Prof. Andrzej Wiśniewski's attainments in the field of erotetic logic, and in particular development of the concept of inferential erotetic logic.

Prof. Wiśniewski, regarded as the father of the Polish school of erotetic logic, over the last three decades has made significant contributions to the logic of questions, proposing and developing inferential erotetic logic.

Erotetics (from the Greek word erotema, meaning "question") is a branch of logic which analyses questions and questioning. Formal logic tools began to be extensively applied to the study of questions in the late 1950s. The first logical theories of questions were developed by various authors, including the Polish logician Tadeusz Kubiński, but these theories differed considerably. New theories arose in the following decades, but there was a lack of consensus on both the general assumptions and specific issues. Prof. Wiśniewski's achievements are regarded as a successful attempt to introduce a new paradigm in erotetic logic.

The inferential logic of questions, or inferential erotetic logic (IEL), for which the Foundation for Polish Science Prize has been awarded, is an idea reaching back to the 1980 s, but it was elaborated by the laureate in the 1990s. Stated most generally, this logic provides formal tools facilitating the modelling of the phenomenon of posing questions in the research process. This is the logic of "erotetic inferences," i.e. inferences in which questions perform the role of conclusions, while the premises are either declarative sentences, or both declarative sentences and questions. Because questions are neither true nor false, valid inferences leading to questions cannot be described using only the conceptual apparatus of the logic of declarative sentences. By introducing some semantic concepts applicable only to questions, IEL characterizes logically valid erotetic inferences. Minimal erotetic semantics (MiES), developed by Prof. Wiśniewski, allows to base the declarative part of IEL either on classical logic or non-classical logics. Besides characterizing the semantic relations underlying the validity of erotetic inferences, MiES also enables strict definitions of many other concepts involving questions, such as soundness of a question, various types of presuppositions of questions, types of answers, and so on.

A vital element of the research process is passing from principal questions to auxiliary questions, and after obtaining answers to them, possibly moving on to further auxiliary questions. The logic of questions proposed by Prof. Wiśniewski offers "erotetic search scenarios," which tell what auxiliary questions should be asked and when they should be asked. Erotetic scenarios are not sets of heuristic rules, but abstract entities having the form of labelled trees, in which the nodes labelled with questions are in the semantic relation underlying logically valid inferences leading from questions to questions.

Although research in erotetic logic might seem on the surface to involve purely theoretical deliberations, it can carry over to practice and new technologies. The 
theories developed by Prof. Wiśniewski may be applied for example in machine learning, the development of artificial intelligence, improvement in internet search engines, and effective analysis of databases. These theories are also applied by other researchers, including in cooperation with Prof. Wiśniewski, for example to analysis of the famous Turing Test, in proof theory, and in modelling of the logical structure of dialogues and arguments.

Dominika Wojtysiak-Łańska The Foundation for Polish Science Programme Promotion and External Cooperation Section 
